\title{
Neutrino-Nucleus Reaction Cross Sections for Neutrino Detection and Nucleosynthesis in Supernova Explosions
}

\author{
Toshio Suzuki ${ }^{1}$ \\ Department of Physics, College of Humanities and Sciences, Nihon University \\ Sakurajosui 3-25-40, Setagaya-ku, Tokyo 156-8550, Japan \\ E-mail:suzuki@phys.chs.nihon-u.ac.jp
}

\section{A. Baha Balantekin}

Department of Physics, University of Wisconsin, Madison, Wisconsin 53706, USA

E-mail: baha@physics.wisc.edu

\section{Toshitaka Kajino}

National Astronomical Observatory of Japan, Mitaka, Tokyo 181-8588, Japan

E-mail: kajino@nao.ac.jp

\section{Satoshi Chiba}

Research Laboratory for Nuclear Reactors, Tokyo Institute of Technology, eguro, Tokyo 152-8550, Japan E-mail: chiba.satoshi@nr.titech.ac.jp

\section{Michio Honma \\ Center for Mathematical Sciences, University of Aizu, Aizu-Walamatsu, Fukushima 965-8580, Japan \\ E-mail: m-honma@u-aizu.ac.jp}

Neutrino-nucleus cross sections relevant to detection of supernova and reactor neutrinos as well as synthesis of elements in supernova explosions are evaluated by shell-model calculations with new shellmodel Hamiltonians, which can describe spin degree's of freedom in nuclei very well. The new Hamiltonians, SFO for p-shell, GXPF1J for pf-shell and VMU (monopole-based universal interaction), have proper tensor components in common, which lead to proper shell-evolutions. We can now reproduce experimental $v$-induced cross sections available, that is, those of ${ }^{12} \mathrm{C}$ and ${ }^{56} \mathrm{Fe}$, and evaluate the cross sections with sound reliability in various light and medium heavy nuclei. Updated $v$-induced cross sections on ${ }^{12} \mathrm{C},{ }^{13} \mathrm{C},{ }^{16} \mathrm{O},{ }^{56} \mathrm{Fe},{ }^{56} \mathrm{Ni}$ and ${ }^{40} \mathrm{Ar}$ are presented and compared to those previously obtained. The cross sections on ${ }^{12} \mathrm{C}$ are applied to study light element synthesis in supernova explosions and neutrino oscillation parameters. Coherent scattering cross sections on ${ }^{12} \mathrm{C}$ and ${ }^{13} \mathrm{C}$ can be good probes of neutron distributions of the nuclei. ${ }^{13} \mathrm{C}$ is an attractive target for the detection of very low energy neutrinos below $10 \mathrm{MeV}$. Accurate evaluations of $v$-induced cross sections are carried out for ${ }^{16} \mathrm{O}$ and ${ }^{40} \mathrm{Ar}$, which are powerful targets for the detection of supernova neutrinos. Cross sections folded over neutrino spectra with and without the oscillation effects are also compared to each other to see how they are sensitive to the oscillation effects.

The 26th International Nuclear Physics Conference

11-16 September, 2016

Adelaide, Australia

\footnotetext{
${ }^{1}$ Speaker

(C) Copyright owned by the author(s) under the terms of the Creative Commons 


\section{Introduction}

Neutrino-nucleus reaction cross sections at neutrino energies below $100 \mathrm{MeV}$ are evaluated by shell-model calculations with new shell-model Hamiltonians, which can describe spin dependent transitions in nuclei very accurately. A new shell-model Hamiltonian for p-shell, SFO [1], is found to be successful in the description of Gamow-Teller (GT) strengths in ${ }^{12} \mathrm{C}$ and ${ }^{14} \mathrm{C}$ as well as magnetic dipole (M1) moments of p-shell nuclei. One for pf-shell, GXPF1J [2], is successful to reproduce the GT strengths in $\mathrm{Ni}$ and Fe isotopes and M1 strengths in pf-shell nuclei. A common feature for these Hamiltonians is the proper incorporation of the spin dependent tensor components in the interactions. The tensor interaction is important to reproduce the vanishing GT strength in ${ }^{14} \mathrm{C}$ [3]. Monopole terms of the tensor forces have a robust sign rule. They are attractive between the different spin-orbit partner orbits $\left(j_{>}=\ell+1 / 2\right.$ and $\mathrm{j}_{<}=\ell-1 / 2$ ), while they are repulsive between the same spin-orbit partner orbits [4]. This sign rule leads to proper shell evolutions toward drip-lines such as the change of magic numbers in neutron-rich nuclei. We have also introduced a monopole-based universal interaction (VMU) [5], which has a simple central and tensor forces and can produce a variety of shell evolutions connecting stable and unstable nuclei. Neutrino-induced reactions are studied with the use of these Hamiltonians, and new cross sections are applied to nucleosynthesis in supernova explosions ( $\mathrm{SNe})$, detection of low energy neutrinos and neutrino oscillation problems.

In sect. 2, $v$-induced cross sections on ${ }^{12} \mathrm{C},{ }^{13} \mathrm{C}$ and ${ }^{16} \mathrm{O}$ are updated by using new p-shell and p-sd shell Hamiltonians, and compared with previous calculations. The cross sections for ${ }^{12} \mathrm{C}$ are applied to synthesis of light elements in SNe both with and without the $v$-oscillation effects. ${ }^{13} \mathrm{C}$ is shown to be a good candidate for the detection of very low energy neutrinos below $10 \mathrm{MeV}$. Coherent scatterings on the carbon isotopes can be good probes of neutron density distributions. Partial cross sections for ${ }^{16} \mathrm{O}$ in various particle and multi-particle emission channels are obtained, and implications on the light element synthesis in $\mathrm{SNe}$ are discussed. In sect. 3, v-induced cross sections on ${ }^{56} \mathrm{Fe},{ }^{56} \mathrm{Ni}$ and ${ }^{40} \mathrm{Ar}$ are discussed with new shell-model Hamiltonians, which can reproduce the Gamow-Teller (GT) strength in the nuclei. The updated neutral current reaction cross sections on ${ }^{56} \mathrm{Ni}$ are shown to lead to enhanced production yield of ${ }^{55} \mathrm{Mn}$ in population III stars. In sect. 4, we investigate if we can identify $v$ spectrum with both collective and Mihailov-Smirnov-Wolfenstein (MSW) resonance oscillations by low energy $v$ scatterings. A summary is given in sect. 5 .

\section{Neutrino-induced reactions on ${ }^{12} \mathrm{C},{ }^{13} \mathrm{C}$ and ${ }^{16} \mathrm{O}$}

We study $v$-induced reactions on ${ }^{12} \mathrm{C}$ and ${ }^{13} \mathrm{C}$ by using the SFO Hamiltonian [1], which can well reproduce the GT strength in ${ }^{12} \mathrm{C}$. Charged-current and neutral-current reaction cross sections for ${ }^{12} \mathrm{C}$ are used to study nucleosynthesis of ${ }^{11} \mathrm{~B}$ and ${ }^{7} \mathrm{Li}$ in $\mathrm{SNe}$. Coherent scatterings on ${ }^{12} \mathrm{C}$ and ${ }^{13} \mathrm{C}$ as probes of neutron distributions are discussed. Spin-dipole strengths in ${ }^{16} \mathrm{O}$ are studied with a modified SFO Hamiltonian [6], and updated $v$-induced reaction cross sections on ${ }^{16} \mathrm{O}$ are used to study synthesis of ${ }^{11} \mathrm{~B}$ and ${ }^{11} \mathrm{C}$ in $\mathrm{SNe}$.

\section{$2.1 \quad v-{ }^{12} \mathrm{C}$ reactions and synthesis of light elements in $\mathrm{SNe}$}


As the GT strength in ${ }^{12} \mathrm{C}$ is well described by shell-model calculations with the use of SFO with p-sd configurations, exclusive charged-current reaction cross sections on ${ }^{12} \mathrm{C}$ reproduce the experimental values very well with $\mathrm{g}_{\mathrm{A}}{ }^{\text {eff }} / \mathrm{g}_{\mathrm{A}}=0.95$ at $\mathrm{E}_{v} \leqq 50 \mathrm{MeV}[7,8]$. Cross sections folded over decay-at-rest (DAR) $v_{\mathrm{e}}$ are also well reproduced. Calculated inclusive reaction cross sections for DAR $v_{\mathrm{e}}$, which include contributions from spin-dipole transitions, are consisitent with the experiment at LSND (see Ref. [7,8]). Exclusive neutral-current reaction cross sections for DAR $v$ also agree well with the experimental data at KARMEN within error bars (see Ref. $[7,8]$ ). These cross sections are improved over conventional shell-model results as well as RPA calculations.

Nucleosynthesis of light elements in SNe are studied by using these improved cross sections for ${ }^{12} \mathrm{C}$ as well as for ${ }^{4} \mathrm{He}[7,9]$. Branching ratios for $\gamma$ and particle emission channels are evaluated by Hauser-Feshbach model, and partial cross sections for each channel are obtained. Production yields of ${ }^{11} \mathrm{~B}$ and ${ }^{7} \mathrm{Li}$ are found to be enhanced compared with previous calculations [10]. When MSW neutrino oscillation effects at He-C layer are taken into account, the yield ratio ${ }^{7} \mathrm{Li} /{ }^{11} \mathrm{~B}$ is found to be enhanced for the nornal neutrino mass-hierarchy while it remains to be unchanged for the inverted mass -hierarchy [7,8,9]. This difference can be used to distinguish the neutrino mass hierarchies. A study of pre-solar grains in Marchison meteorite lead to a conclusion that the inverted hierarchy is statistically more favored [11].

\section{$2.2 v-{ }^{13} \mathrm{C}$ reactions}

We now discuss $v-{ }^{13} \mathrm{C}$ reactions. This nucleus is an attractive target for very low energy neutrinos below $10 \mathrm{MeV}$ such as reactor neutrinos. Since the threshold energy for $v-{ }^{12} \mathrm{C}$ is about $13 \mathrm{MeV}$, low energy $v$ with $\mathrm{E}_{v} \leq 10 \mathrm{MeV}$ can excite only ${ }^{13} \mathrm{C}$, which consitutes $1.07 \%$ of natural carbon. Cross sections for GT and Fermi transitions to low lying sates in ${ }^{13} \mathrm{~N}$ and ${ }^{13} \mathrm{C}$ are evaluated with SFO, which are found to be larger than those obtained with the CohenKurath intearction within p-shell [12].

Partial cross sections for $\gamma$ and particle emissions are obtained with the HauserFeshbach theory. In neutral-current reactions, coherent elastic scattering has the largest cross section, and next largest branch is $\gamma$ emission. Neutron emission channels have also rather large cross sections due to a small neutron threshold energy. Among them the transition to the ground state of ${ }^{12} \mathrm{C}$ is dominant, but the transition to ${ }^{12} \mathrm{C}\left(2^{+}, 4.44 \mathrm{MeV}\right)$ followed by $\gamma$ emission has a sizable measurable contribution. Simultaneous measurement of $\gamma$ and neutron can be a good probe for the detection of very low energy neutrinos.

Here, we comment on coherent elastic scatterings on the carbon isotopes. Neutral-current has the following structure with axial-vector and vector components, $\mathrm{J}_{\mu}{ }^{(0)}=\mathrm{A}_{\mu}{ }^{3}+\mathrm{V}_{\mu}{ }^{3}$ $-2 \sin ^{2} \theta_{\mathrm{W}} \mathbf{J}_{\mu}{ }^{\gamma}$, where $\mathrm{A}_{\mu}{ }^{3}\left(\mathrm{~V}_{\mu}{ }^{3}\right)$ is the weak axial (vector) current, $\mathbf{J}_{\mu}{ }^{\gamma}$ is the electromagnetic current and $\theta_{\mathrm{w}}$ is the Weinberg angle. For the elastic scatterings, the dominant contributions come from the $0^{+}$multipole induced by the vector current part, $\left(\mathrm{G}_{\mathrm{E}}{ }^{\mathrm{IV}}-2 \sin ^{2} \theta{ }_{\mathrm{w}} \mathrm{G}_{\mathrm{E}}\right)$. $\left\langle\right.$ g.s. $\left|\mathrm{j}_{0}(\mathrm{qr}) \mathrm{Y}^{(0)}\right|$ g.s. $>$, though the $1^{+}$multipole term also contributes in case of ${ }^{13} \mathrm{C}$. Here, $\mathrm{G}_{\mathrm{E}}$ is the electric form factor of nucleon and $\mathrm{G}_{\mathrm{E}}{ }^{\mathrm{IV}}$ is its isovector part. The nuclear form factor probes mostly the neutron distribution of the nucleus; $\mathrm{G}_{\mathrm{E}}^{\mathrm{p}}\left(1-4 \sin ^{2} \theta_{\mathrm{w}}\right) / 2 \cdot \rho_{\mathrm{p}}(\mathrm{r})-\mathrm{G}_{\mathrm{E}}^{\mathrm{p}} \cdot \rho_{\mathrm{n}}(\mathrm{r})$. $\mathrm{G}_{\mathrm{E}}{ }^{\mathrm{p}}$ is the electric form factor of proton and $\mathrm{G}_{\mathrm{E}}{ }^{\mathrm{n}}$ (that of neutron) was neglected. Neutron and 
proton density distributions are denoted by $\rho_{\mathrm{n}}$ and $\rho_{\mathrm{p}}$, respectively. As $\sin ^{2} \theta_{\mathrm{W}}=0.23$, the first term from the proton is quite small, and the second term from the neutron is dominant. Thus, the neutral-current coherent elastic scatterings can probe the neutron density distributions in nuclei [13]. As carbon isotopes are light-mass nuclei, measurement of the cross sections by recoil nuclei is more accessible than the case of heavier nuclei such as xenon.

\section{$2.3 \quad v-{ }^{16} \mathrm{O}$ reactions}

We next study $v-{ }^{16} \mathrm{O}$ reactions. The ${ }^{16} \mathrm{O}$ nucleus is contained in water, the important target for neutrinos, and precise evaluations of reaction cross sections are quite important. Main contributions to the cross sections come from spin-dipole transitions. A modified SFO Hamiltonian - SFO-tls [6], whose p-sd cross shell matrix elements have tensor components of $\pi+\rho$ meson exchanges and two-body spin-orbit components of $\sigma+\omega+\rho$ meson exchanges, is used to obtain spin-dipole transition strengths and charged-current and neutral-current reaction cross sections. The strengths are shifted toward lower excitation energies compared with those of SFO, and the cross sections are enhanced compared with SFO as well as CRPA calculations [14] as shown in Fig. 1.
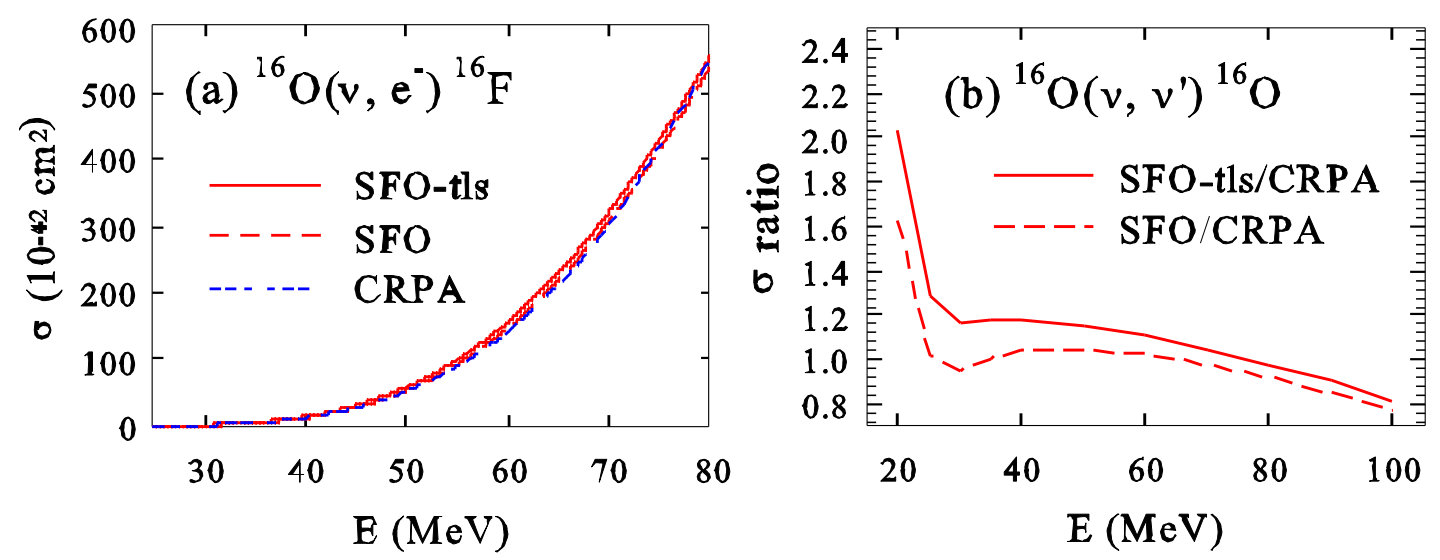

Fig. 1 (a) Charged-current reaction cross sections for ${ }^{16} \mathrm{O}$ obtained with SFO-tls [6], SFO [1] and CRPA [14]. (b) Ratios of the cross sections of SFO-tls and SFO compated to that of CRPA.

Branching ratios for particle and multi-particle emissions are obtained by HauserFeshbach model. Besides proton, neutron, d, pn and pp emissions, a sizable branching ratio for $\alpha \mathrm{p}$ emission channel is found in the neutral-current reaction. The cross section for ${ }^{16} \mathrm{O}(v, v$, $\alpha p){ }^{11} \mathrm{~B}$ is as large as about $20 \%$ of the cross section for ${ }^{12} \mathrm{C}(v, v ' \mathrm{p}){ }^{11} \mathrm{~B}$. This gives rise to an additional production of ${ }^{11} \mathrm{~B}$ from $v-{ }^{16} \mathrm{O}$ reactions in $\mathrm{SNe}$. The production yield of ${ }^{11} \mathrm{~B}$ is found to be enhanced by about $7 \%$ and $17 \%$ in $\mathrm{SNe}$ of stars of masses with $15 \mathrm{M}_{\odot}$ and $20 \mathrm{M}_{\odot}$, respectively, when multi-particle emission channels such as $\alpha \mathrm{p}$ emission are included in addition to $\gamma, \mathrm{n}, \mathrm{p}$ and $\alpha$ emission channels [15].

\section{Neutrino-induced reactions on ${ }^{56} \mathrm{Fe},{ }^{56} \mathrm{Ni}$ and ${ }^{40} \mathrm{Ar}$}


Now we discuss neutrino-nucleus reactions on pf-shell nuclei and a sd-pf shell nucleus, ${ }^{40} \mathrm{Ar}$. First, we discuss ${ }^{56} \mathrm{Fe}$ as it is one of a very few targets for which there exist experimental data. Neutrino-induced reaction cross sections on ${ }^{56} \mathrm{Fe}$ are evaluated with a new shell-model Hamiltonian for pf-shell, GXPF1J [2], and compared with the experimental data as well as previous calculations. Next, we discuss ${ }^{56} \mathrm{Ni}$ as GXPF1J reproduces very well the observed GT strength in ${ }^{56} \mathrm{Ni}$ [16]. Two-peak structure of the distribution of the GT strength is shown to enhance the proton emission channel in the neutral-current reaction on ${ }^{56} \mathrm{Ni}$. Finally, we discuss ${ }^{40} \mathrm{Ar}$ which is a very powerful target for neutrino detections. The GT strength and charged-current reaction cross section are evaluated with the use VMU.

\section{1 $v-{ }^{56} \mathrm{Fe}$ reactions}

Experimental data are available for ${ }^{56} \mathrm{Fe}\left(v_{\mathrm{e}}, \mathrm{e}^{\mathrm{e}}\right){ }^{56} \mathrm{Co}$ for DAR neutrinos [17]. GT strength and Fermi transition strength to the isobaric analog state are obtained by shell-model calculations with GXPF1J [2] and KB3G [18]. We adopt a hybrid method, that is, other multipoles are evaluated by RPA calculations with SGII interaction [19]. Calculated total cross section for GXPF1J+SGII (KB3G+SGII) is $259(255) \times 10^{-42} \mathrm{~cm}^{2}$ [20], which is very close to the experimental value, $(256 \pm 108 \pm 43) \times 10^{-42} \mathrm{~cm}^{2}$ [17] and a result of RPA calculation, 240 $\times 10^{-42} \mathrm{~cm}^{2}$ [21]. We can now reproduce the observed data for ${ }^{56} \mathrm{Fe}$ as well as for ${ }^{12} \mathrm{C}$ (sect. 2.1).

\section{2 $v-{ }^{56} \mathrm{Ni}$ reactions and synthesis of ${ }^{55} \mathrm{Mn}$}

The GXPF1J Hamiltonian can describe GT strengths in $\mathrm{Ni}$ isotopes very well. In particular, the two-peak structure in the GT strength in ${ }^{56} \mathrm{Ni}$ observed by (p, n) reaction [16] is reproduced quite nicely by GXPF1J while it is not possible by other conventional shell-model Hamiltonians such as KB3G. In the neutral-current reaction, ${ }^{56} \mathrm{Ni}\left(v, v^{\prime}\right){ }^{56} \mathrm{Ni}$, ptoton emission channel effectively opens just from the beginning of the second peak of the GT strength in ${ }^{56} \mathrm{Ni}$. This leads to an enhancement of the cross section for ${ }^{56} \mathrm{Ni}\left(v, v\right.$ 'p) ${ }^{55} \mathrm{Co}$ reaction. ${ }^{55} \mathrm{Mn}$ is produced after successive e-capture reactions on ${ }^{55} \mathrm{Co}$ in population III stars. Production yield of ${ }^{55} \mathrm{Mn}$ is enhanced due to the enhancement of the proton-emission cross sections for GXPF1J by the $v$-process nucleosynthesis [20].

\section{3 $v{ }^{40} \mathrm{Ar}$ reactions}

Liquid ${ }^{40} \mathrm{Ar}$ is a powerful target for the detection of supernova neutrinos and studies of $v$ oscillations. GT strength and charged-current reaction cross sections on ${ }^{40} \mathrm{Ar}$ are evaluated by shell-model calculations with the use VMU [5]. For sd-shell SDPF-M Hamiltonian [22] is used while GXPF1J is used for pf-shell. The sd-pf cross-shell matrix elements are constructed from VMU with two-body spin-orbit forces. The GT strength in ${ }^{40} \mathrm{Ar}$ obtained by $(\mathrm{p}, \mathrm{n})$ reaction [23] is well described by the present interaction. Previous GT strength in Ref. [24] is smaller than the present calculated strength as well as the experimental data. Cross sections for ${ }^{40} \mathrm{Ar}\left(v, \mathrm{e}^{-}\right){ }^{40} \mathrm{~K}$ are evaluated by shell-model calculations for $1^{+}$and $0^{+}$transitions and RPA calculations for other multipoles [25]. The GT and Fermi contributions of the present work are found to be larger than those of shell-model calculations in Ref. [24] by about $25 \%$ and RPA calculations in Ref. [26] by about $40 \%$. 


\section{Identification of $v$ spectrum with neutrino oscillations}

We address a problem how we can distinguish neutrino spectra with $v$ oscillations and without $v$ oscillations. We investigate here what differences may arise for $v_{\mathrm{e}}$-induced reaction cross sections when supernova neutrino spectra are affected by neutrino oscillations, both collective and MSW oscillations. When collective oscillations occur near the center of the SNe, electron neutrinos and heavy-flavor neutrinos $\left(v_{\mathrm{x}}\right)$ swap each other at $\mathrm{E}_{v} \geq \mathrm{E}_{\text {split }}$ in case of inverted hierarchy [27]. The split energy, $\mathrm{E}_{\text {split }}$, is about $\mathrm{E}_{v}=8 \mathrm{MeV}$. The reaction cross sections are expected to be enhanced for the inverted hierarchy as heavy-flavor neutrinos are produced with higher energies than electron neutrinos, while they remain unchanged for the normal hierarchy. Then, the high-density MSW oscillations take place in case of the normal hierarchy, which swaps the spectra of electron and heavy-flavor neutrinos. In case of the inverted hierarchy, low-density MSW oscillation affects the $v_{\mathrm{e}}$ spectrum at $\mathrm{E}_{v} \leq \mathrm{E}_{\text {split }}$.

The electron neutrino spectra after both collective and MSW oscillation effects are expressed as follows [27]:

$$
\mathrm{S}_{v}(\mathrm{E})=\mathrm{S}_{-}\left\{v_{\mathrm{x}}\right\}(\mathrm{E})
$$

for the normal mass hierarchy, and

$$
\begin{aligned}
& \mathrm{S}_{v}(\mathrm{E})=\sin ^{2} \theta_{12} \mathrm{~S}_{-}\left\{v_{\mathrm{e}}\right\}(\mathrm{E})+\cos ^{2} \theta_{12} \mathrm{~S}_{-}\left\{v_{\mathrm{x}}\right\}(\mathrm{E}) \quad \text { at } \mathrm{E}<\mathrm{E}_{\text {split }} \\
& \mathrm{S}_{v}(\mathrm{E})=\mathrm{S}_{-}\left\{v_{\mathrm{x}}\right\}(\mathrm{E}) \quad \text { at } \mathrm{E}>\mathrm{E}_{\text {split }}
\end{aligned}
$$

for the inverted mass hierarchy, where $S_{-}\left\{v_{\mathrm{e}}\right\}(\mathrm{E})$ and $\mathrm{S}_{-}\left\{v_{\mathrm{x}}\right\}(\mathrm{E})$ are neutrino spectra of $v_{\mathrm{e}}$ and $v_{\mathrm{x}}$, respectively, which are taken to be Fermi-Dirac distribution with temperatures that give averaged energies of $\left\langle\mathrm{E}_{-}\left\{v_{\mathrm{e}}\right\}\right\rangle=10 \mathrm{MeV}$ and $\left\langle\mathrm{E}_{-}\left\{v_{\mathrm{x}}\right\}\right\rangle=18 \mathrm{MeV}$.

\begin{tabular}{|l|c|c|}
\hline${ }^{13} \mathrm{C}\left(v_{\mathrm{e}}, \mathrm{e}^{-}\right){ }^{13} \mathrm{~N}$ & Normal hierarchy & Inverted hierarchy \\
\hline No oscillations & 8.01 & 8.01 \\
\hline Collectiv oscillations & 8.01 & 39.44 \\
\hline Collctive + MSW oscillations & 39.31 & 39.35 \\
\hline \hline${ }^{48} \mathrm{Ca}\left(v_{\mathrm{e}}, \mathrm{e}^{-}\right){ }^{48} \mathrm{Sc}$ & Normal hierarchy & Inverted hierarchy \\
\hline No oscillations & 73.56 & 73.56 \\
\hline Collectiv oscillations & 73.56 & 303.4 \\
\hline Collctive + MSW oscillations & 302.6 & 302.8 \\
\hline
\end{tabular}

Table I Reaction cross sections for ${ }^{13} \mathrm{C}\left(v_{\mathrm{e}}, \mathrm{e}^{-}\right){ }^{13} \mathrm{~N}$ and ${ }^{48} \mathrm{Ca}\left(v_{\mathrm{e}}, \mathrm{e}^{-}\right){ }^{48} \mathrm{Sc}$ in units of $10^{-42} \mathrm{~cm}^{2}$ folded over the neutrino spectra modified by collective and MSW oscillations denoted by Eqs. (1) and (2)

Calculated cross sections for ${ }^{13} \mathrm{C}\left(v_{\mathrm{e}}, \mathrm{e}^{-}\right){ }^{13} \mathrm{~N}$ and ${ }^{48} \mathrm{Ca}\left(v_{\mathrm{e}}, \mathrm{e}^{\mathrm{e}}\right){ }^{48} \mathrm{Sc}$ folded over the spectra are shown in Table I. The targets ${ }^{13} \mathrm{C}$ and ${ }^{48} \mathrm{Ca}$ are chosen as these nuclei have large GT strengths at low excitation energies, which is favorable to distinguish the spectra at low energies, $\mathrm{E}_{v} \leq \mathrm{E}_{\text {split. }}$ As we see from Table I, the mass hierarchies can be distinguished if only the collective oscillations occur. However, if both collective and MSW oscillation effects are taken into account, the difference of the cross sections are too small to distinguish the hierarchies. This is due to a small split enegy $\mathrm{E}_{\text {split }} \approx 8 \mathrm{MeV}$. The difference of the spectra below $\mathrm{E}_{\text {split }}$ is 
difficult to distinguish as the reaction cross sections are proportional to $\mathrm{E}_{v}{ }^{2}$. If the collective oscillations give rise to differences in element synthesis in $\mathrm{SNe}$, we may be able to find a way to distinguish the mass hierarchies. The model of swapping of spectra by collective excitations adopted here is one for two-flavor neutrino case. It would be interesting to extend the model to theree-flavor case.

\section{Summary}

New shell-model Hamiltonians, SFO for p-shell, GXPF1J for pf-shell and VMU (monopole-based universal interaction), which have proper tensor components and describe spin modes of nuclei quite accurately, are used to evaluate neutrino-nucleus reaction cross sections. We have now come to be able to accurately evaluate $v$-induced cross sections in light and medium-heavy nuclei. Updated $v$-induced cross sections are obtained for ${ }^{12} \mathrm{C},{ }^{13} \mathrm{C},{ }^{16} \mathrm{O},{ }^{56} \mathrm{Fe},{ }^{56} \mathrm{Ni}$ and ${ }^{40} \mathrm{Ar}$. Light element synthesis such as ${ }^{11} \mathrm{~B}$ in $\mathrm{SNe}$ is discussed with the use of new cross sections for ${ }^{12} \mathrm{C}$ and ${ }^{16} \mathrm{O}$. New cross sections for ${ }^{56} \mathrm{Ni}$ are used for nucleosynthesis of ${ }^{55} \mathrm{Mn}$ in population III stars. More reliable cross sections are obtained for ${ }^{13} \mathrm{C}$ and ${ }^{40} \mathrm{Ar}$, which are powerful targets for detection of reactor and supernova neutrinos. Coherent scattering on carbon isotopes are shown to be good probes of neutron density distributions in the nuclei. The MSW oscillation effects in SNe are pointed out to be useful to distingush the $v$ mass hierarchies. A case in which both collective and MSW oscillations occur in SNe is also discussed. The split energy for the swapping of spectra in the collective oscillations is too small to distingush the mass hierarchies from the differences of $v$-induced cross sections on ${ }^{13} \mathrm{C}$ and ${ }^{48} \mathrm{Ca}$.

This work was supported by Grants-in-Aid for Scientific Research (15K05090) of the MEXT of Japan.

\section{References}

[1] T. Suzuki, R. Fujimoto and T. Otsuka, Gamow-Teller transitions and magnetic properties of nuclei and shell evolution, Phys. Rev. C 67 (2003) 044302.

[2] M. Honma et al., Effective interaction for pf-shell nuclei, Phys. Rev. C 65 (2002) 061301 (R);

M. Honma et al., Effective interaction for nuclei of A=50-100 and Gamow-Teller properties, J. Phys. Conf. Ser. 20 (2005) 7.

[3] I. Talmi, Fifty Years of the Shell Model - The Quest for the Effective Interaction, Adv. in Nucl. Phys. 27 (2003) 1.

[4] T. Otsuka, T. Suzuki, R. Fujimoto, R. Grawe and Y. Akaishi, Evolution of Nuclear Shells due to the Tensor Force, Phys. Rev. Lett. 95 (2005) 232502.

[5] T. Otsuka, T. Suzuki, M. Honma, Y. Utsuno, N. Tsunoda, Novel Features of Nuclear Forces and Shell Evolution in Exotic Nuclei, Phys. Rev. Lett. 104 (2010) 012501.

[6] T. Suzuki and T. Otsuka, Exotic Magnetic Properties in ${ }^{17}$ C, Phys. Rev. C 78 (2008) 061301 (R).

[7] T. Suzuki, S. Chiba, T. Yoshida, T. Kajino and T. Otsuka, Neutrino-nucleus reactions based on new shell-model Hamiltonians, Phys. Rev. C 74 (2006) 034307.

[8] T. Suzuki and T. Kajino, Element synthesis in the supernova environment and neutrino oscillations, J. Phys. G 40 (2013) 083101. 
[9] T. Yoshida, T. Suzuki et al., Neutrino-nucleus reaction cross sections for light element synthesis in supernova explosions, The Astrophys. J. 686 (2008) 448.

[10] S. E. Woosley et al., The v-process, The Astrophys. J. 356 (1990) 272.

[11] G. J. Mathews, T. Kajino, W. Aoki, W. Fujiya and J. B. Pitts, Exploring the neutrino mass hierarchy probability with meteoritic supernova material, $v$-process nucleosynthesis, and $\theta_{13}$ mixing, Phys. Rev. D 85 (2012) 105023.

[12] T. Suzuki, A. B. Balantekin and T. Kajino, Neutrino capture on ${ }^{13}$ C, Phys. Rev. C 86 (2012) 015502.

[13] K. Patton et al., Neutrino-nucleus coherent scattering as a probe of neutron density distributions, Phys. Rev. C 86 (2012) 024612.

[14] E. Kolbe, K. Langanke and P. Vogel, Estimates of weak and electromagnetic nuclear decay signatures for neutrino reactions in Super-Kamiokande, Phys. Rev. D 66 (2002) 013007.

[15] T. Suzuki, S. Chiba and T. Yoshida, to be published.

[16] M. Sasano et al. , Gamow-Teller Transition Strengths from ${ }^{56}$ Ni, Phys. Rev. Lett. 107 (2011) 202501.

[17] R. Maschuw (KARMEN collaboration), Neutrino spectroscopy with KARMEN, Prog. Part. Nucl. Phys. 40 (1998) 183.

[18] N. Van Giai and H. Sagawa, Spin-isospin and pairing properties of modified Skyrme interactions, Phys. Lett. B 106 (1981) 379.

[19] E. Caurier, G. Martinez-Pinedo, F. Nowacki, A. Poves and A. P. Zuker, The shell model as a unified view of nuclear structure, Rev. Mod. Phys.77 (2005) 427.

[20] T. Suzuki, M. Honma et al., Neutrino induced reactions on ${ }^{56} \mathrm{Fe}$ and ${ }^{56} \mathrm{~N}, \mathrm{i}$ and production of ${ }^{55} \mathrm{Mn}$ in population III stars, Phys. Rev. C 79 (2009) 061603 (R).

[21] E. Kolbe and K. Langanke, Role of $v$-induced reactions on lead and iron in neutrino detectors, Phys. Rev. C 63 (2001) 025802.

[22] Y. Utsuno, T. Otsuka, T. Mizusaki and M. Honma, Varing shell gap and deformation in N 20 unstable nuclei studied by the Monte Carlo shell model., Phys. Rev. C 60 (1999) 054315.

[23] M. Bhattacharya, C. D. Goodman and A. Garcia, Weak-interaction strength from charge-exchange reactions versus $\beta$ decay in the $A=40$ isoquintet, Phys. Rev. C 80 (2009) 055501

[24] W. E. Ormand, P. M. Pizzochero, P. F. Bortignon and R. A. Broglia, Neutrino capture cross sections for ${ }^{40}$ Ar and $\beta$-decay of ${ }^{40}$ Ti, Phys. Lett. B 345 (1995) 343.

[25] T. Suzuki and M. Honma, Neutrino capture recations on ${ }^{40}$ Ar, Phys. Rev. C 87 (2013) 014607.

[26] E. Kolbe, K. Langanke, G. Martinez-Pinedo and P. Vogel, Neutrino-nucleus reactions and nuclear structure, J. Phys. G 29 (2003) 2569.

[27] G. G. Raffelt, Physics opportunities with supernova neutrinos, Prog. Part. Nucl. Phys. 64 (2010) 393. 